\title{
Seroprevalence of HCV, HBV, HIV and syphilis among blood donors at Beni-Seuf University Hospital blood bank
}

\author{
Original \\ Nashaat N. Ismail', Rashad M. Mostafa', Maha A. Fouad ${ }^{3}$ \\ Article \\ ${ }^{1,3}$ Department of Andrology and S.T.Ds, Faculty of Medicine, Beni-Seuf University \\ ${ }^{2}$ Department of Andrology, Dermatology and Venereology, Faculty of Medicine, Suez \\ Canal University
}

\begin{abstract}
Introduction: Hepatitis B virus (HBV), hepatitis C virus (HCV), human immunodeficiency virus (HIV) and syphilis are the serious infections transmitted during blood transfusion especially with blood donation put millions of people at risk of transfusion-transmissible infections (TTIs).

Purpose:To determine the seroprevalence of hepatitis B surface antigen, hepatitis C virus antibody, anti-human immunodeficiency virus, and syphilis in blood donors in Beni-Seuf University hospital blood bank.

Materials and Methods:This was a retrospective, descriptive study. All blood donors' records from November 2007 to April 2015 were included. We analyzed data from 30055 blood samples collected. ELISA technique to detect HCV Ab, HBsAg, $\mathrm{HIV} \mathrm{Ab}$ and VDRL for syphilis was used.

Results: Seroprevalence of HCV, HBV, HIV and syphilis among blood donors were (9.4, 1.6, 0.1 and 0.0002, respectively). The majority of donors, 28197 (93.3\%) were males, while females were 1858 (6.7\%). Prevalence of HBsAg and anti-HCV were significantly higher among males $(1.5 \%, 8.8 \%$, respectively) than females $(0.1 \%$ and $0.6 \%$, respectively $)$ with $(P<0.001)$. There was a decreasing prevalence of HCV among blood donors as the annual Anti-HCV prevalence dropped significantly from 3.29 in 2007 to $0.4 \%$ in 2015 with $(P<0.01)$, whereas there was a mild variation in the prevalence of $\mathrm{HBs} A g$, throughout the study period, that was not statistically significant $(P=0.6)$.

Conclusion: It is important to continue screening donated blood with highly sensitive and specific tests to ensure the safety of blood for recipient.
\end{abstract}

Key Words: AIDS, HBV, HCV, transfusion-transmitted infections, syphilis

Received: 17 June 2017, Accepted: 28 October 2017

Corresponding Author: Rashad Mahmoud Mostafa, Assistant professor of Andrology, Sexology and STDs, Andrology Unit, Faculty of Medicine, Suez-Canal University, Ismailia, Egypt Tel.: 002-0101393823, E-mail: rashad1010@yahoo.com

ISSN: 2090-6048, March 2018, Vol. 8, No. 1

\section{INTRODUCTION}

Blood donation is an important procedure that saves millions of lives; however, unsafe transfusion practices also put millions of people at risk of transfusion-transmissible infections (TTIs) ${ }^{[1]}$. An unsafe blood transfusion is very costly from both economic and human points of view. Long-term morbidity and mortality, delayed viraemia and hidden states resulting from the transfusion of infected blood have far-reaching consequences, not only for the recipients themselves, but also for their families and their communities $^{[3]}$. A number of viruses, bacteria and parasites can be transmitted through blood or blood products. Hepatitis B virus (HBV), hepatitis C virus (HCV), human immunodeficiency virus (HIV) and syphilis are the most serious infections transmitted during blood transfusion ${ }^{[2]}$. $\mathrm{HBV}, \mathrm{HCV}, \mathrm{HIV}$ and syphilis infections are common serious complications of blood transfusion. Prevention of TTIs in developed countries has been achieved by reducing unnecessary transfusions, using only regular voluntary donors, excluding donors with specific risk factors and systematic screening of all donated blood for infection. By contrast, in many developing countries none of these interventions is applied uniformly and the risk of transfusion-transmitted infections remains high ${ }^{[4]}$. Despite progress in the diagnosis and treatment of viral hepatitis, their incidence is still high in some parts of the world. In the context of globalization, which currently facilitates the large-scale spread of disease more than ever, all regions are exposed to the risk of viral infections ${ }^{[5]}$. To prevent TTIs, mandatory screening tests were performed on the blood before transfusion for HIV, HBV, HCV and syphilis by blood transfusion centers in Egypt ${ }^{[6]}$. Estimating the prevalence of TTIs; namely, HBV, HCV, HIV and syphilis, among blood donors can reveal the problem of unnoticeable infections in healthy-looking members of the general population and also provide data that is important in formulating the strategies for improving the management of a safe blood supply. In addition, it can give us a guide to the magnitude of some sexually transmitted infections in the community ${ }^{[6]}$. 


\section{PATIENTS AND METHODS}

\section{Aim of the work:}

To determine the seroprevalence of hepatitis B surface antigen (HBsAg), hepatitis $\mathrm{C}$ virus antibody (anti-HCV), anti-human immunodeficiency virus (anti-HIV), and VDRL among blood donors in Beni-Seuf University Hospital blood bank.

\section{Subjects and Methods:}

This study was a retrospective, descriptive study conducted on 30055 blood donors in blood bank of BeniSeuf university Hospital from the period of November 2007 to April 2015.

\section{Methods:}

The data were recorded in a specially designed data collection form of blood-bank of Beni-Seuf University Hospital which included the following information:

\section{(A) Donors information:}

Blood bank serial number, age, sex, residence: donors from Beni-Suef Government or outside government, the year of data collection, blood group of donor and blood pressure during donation.

\section{(B) Laboratory investigation:}

\section{1- General: Complete Blood Count.}

2-Specific: ELISA technique was used to detect HBsAg, $\mathrm{HCV} \mathrm{Ab}, \mathrm{HIV} \mathrm{Ab}$ and syphilis anti- body.

\section{For Hepatitis $B$ virus detection:}

ELISA technique (Monalisa HBsAg ULTRA) was used to detect HBsAg in sample of blood bank donors. Distribution of control sera and samples into wells of microplate. This distribution can be visually controlled as there is a difference of coloration between empty well and well with sample. This distribution can be controlled automatically by reading at 490700-620/ $\mathrm{nm}$. Distribution of red coloured conjugate into wells also can be visually controlled. The sample deposition can be controlled by automatic reading at 490700-620/ $\mathrm{nm}$. After incubation at $37{ }^{\circ} \mathrm{C}$ during one hour and half the unbound conjugate is removed by washing. Distribution of coloured substarte solution can be visually controlled as there is a clear difference of colouration between empty well and well with pink substrate solution, this distribution can also be controlled automatically by reading at $490 \mathrm{~nm}$. After 30 minutes incubation in presence of substrate in dark and at room temperature $\left(1830-{ }^{\circ} \mathrm{C}\right)$. The presence of complexed conjugate is shown by a change of colour. Distribution of stopping solution can be visually controlled: the substarte solution which initially pink becomes uncoloured for the non-reactive sample wells and turn blue to yellow for positive sample wells. Reading of optical densities at $450700-620 / \mathrm{nm}$ and interpretation of the results.

\section{Specimens Preparation:}

a- Collection of blood sample.

b-The test should be performed on undiluted serum or plasma (collected with EDTA, heparin, citrate, ACD-based anticoagulants).

c- Separate the serum or plasma from the clot or red cells as soon as possible to avoid any hemolysis, extensive hemolysis may affect test, suspended fibrin particles or aggregates may yield falsely positive results.

$\mathrm{d}$-The specimen can be stored at $+28-^{\circ} \mathrm{C}$ if screening is performed within 7 days or they may be deep-frozen at $-20^{\circ} \mathrm{C}$ for several months.

e- Avoid repeated freeze/thaw cycles. Samples that have been frozen and defrozen more than 3 times cannot be used. f-Avoid usage of hemolysis, contaminated or hyperglycemic sera or plasma.

\section{For Hepatitis $C$ virus detection:}

By ELISA technique to detect $\mathrm{HCV} \mathrm{Ab}$ in samples of blood bank donors. Microplates are coated with HCVspecific antigens derived from "core" and "ns" regions encoding for conservative and immunodominant antigenic determinants (core peptide, recombinant NS3, NS4, and NS5 peptides). Ab are captured, if present by antigens. After washing out all the other components of the sample, in the $2^{\text {nd }}$ incubation bound $\mathrm{HCV}$ antibodies, IgG and IgM as well, are detected by addition of polyclonal specific anti hIg $\mathrm{G}$ and $\mathrm{M}$ antibodies labeled with peroxidase (HRP). The enzyme captured on the solid phase, acting on the substrate /chromogen mixture, generates an optical signal that is proportional to amount of anti $\mathrm{HCV}$ antibodies present in sample. e- A cut-off value let optical denities be interpreted into $\mathrm{HCV}$ antibody negative and positive results.

\section{Specimen preparation :}

a-Blood is draw a septically by venupuncture and plasma or serum is prepared using standard techniques of preparation of samples for clinical laboratory analysis.

b- Avoid any addition of preservatives to samples ; especially sodium azide as this chemical would affect enzymatic activity of conjugate, generating false negative results.

c- Samples have to be clearly identified with codes on names in order to avoid misinterpretation of results.

d-Hemolysed and hyperlipemic samples have to be discarded as they could generate false results.

e- Sera and samples can be stored at $+2-8^{\circ} \mathrm{C}$ for up to seven days after collection. For longer storage periods, 
samples can be stored frozen at $-20^{\circ} \mathrm{C}$ for several months. Any frozen samples should not be frozen/thawed more than once as this generate particles that could affect results. f- If particles are present , centrifuge at $2.000 \mathrm{rpm}$ for 20 min or filter using 0.20 .8 - $u$ filters to clean up the samples for testing.

\section{For HIV detection:}

ELISA technique to detect $\mathrm{HIV} \mathrm{Ab}$ in samples of blood bank donors and positive samples could be confirmed by Western blot. Synthetic peptides representing immunodominant epitopes of HIV-1 and HIV-2 together with a monoclonal antibody to p24 HIV-1 antigen are coated onto wells of a microplate. The peptide and antibody have been carefully selected to ensure the screening of antibody and p24 antigen to all HIV-1 subtypes, including subtype $\mathrm{O}$ and HIV-2. Serum or plasma samples are added to these wells and, if antibodies specific to HIV-1 and/or HIV-2 (IgG, IgM ,or IgA ) are present in samples, they will form stable complexes with the HIV peptide antigens in the well. In case of HIV-1 p24 is present in the sample ,the antigen will be captured by specific monoclonal antibody. Antigen- antibody complexes are the identified through the successive addition of : 1) biotinylated monoclonal peptides, a biotinylated monoclonal antibody to HIV1p24 and 2) horseradish peroxidase HRP Streptavidin conjugate. e- The hydrolytic activity oh horseradish peroxidase allows for the quantification of these antibodyantigen complexes, then peroxidase substrate solution is added. During incubation a blue colour will develop in proportion to the amount of anti-HIV12/ antibodies or HIV-1 p24 antigen bound to the well, thus establishing their presence or absence in the sample. g- Wells containing samples negative for anti-HIV antibody and/or p24 antigen remains colorless. h- A stop solution is added to each and the resulting yellow color is read on a microplate reader at $450 \mathrm{~nm}$.

\section{Specimen preparation:}

1-Blood is drawn aseptically by venupuncture and plasma or serum is prepared using standard techniques of preparation of samples for clinical laboratory analysis. No influence has been observed in preparation with citrate, EDTA and heparin.

2-Avoid any addition of preservatives to samples, especially sodium azide as this chemical would affect the enzymatic activity of conjugate, generating false negative results. 3-Samples have to be clearly identified with codes on names in order to avoid misinterpretation of results. When the kit is used for screening of blood units, bar code labeling and electronic reading is strongly recommended. 4-Heamolysed or hyperlipemic samples have to be discarded as they could generate false results. Samples containing residues of fibrin or heavy particles or microbial filaments and bodies should be discarded as they could rise to false results.
5-Sera and plasma can be stored at $+2-8^{\circ} \mathrm{C}$ for up to seven days after collection. For longer storage periods, samples can be stored frozen at $-20^{\circ} \mathrm{C}$ for several months. Any frozen samples should not be frozen/thawed more than once as this may generate particles that could affect the test result.

6-If particles are present filter using 0.2- $0.8 \mathrm{u}$ filters to clean up the sample for testing.

7-Do not use heat inactivated samples as they could give false reactivity.

\section{For syphilis detection:}

ELISA technique to detect syphilis antibody in blood bank donor (VDRL). Microplates are coated with purified Treponema pallidum synthetic antigens. Patients serum/ plasma is added to micowell together with a mix of TP synthetic antigens, labelled with peroxidase (HRP). The specific immunocomplex, formed in the presence of anti$\mathrm{TP} \mathrm{Ab}$ in the sample, is captured by the solid phase. At the end of the one-step incubation, microwells are washed to remove unbound serum proteins and HRP conjugate. The chromogen/substrate is then added and in the presence of captured immunocomplex, the colorless substrate is hydrolyzed by the bound HRP conjugate to a colored end product. After blocking the enzymatic reaction, its optical density is measured by an ELISA reader. The color intensity is proportional to amount of anti $\mathrm{TP} \mathrm{Ab}$ present in sample.

\section{Sample preparation:}

1-Blood is drawn a septically by venepuncture and plasma or serum is prepared using standard technique of preparation. No influence has been observed in preparation of the sample with citrate, EDTA and heparin.

2-Avoid any addition of preservatives to samples; especially sodium azides as this chemical would affect the enzymatic activity of conjugate, generating false negative results.

3-Samples have to be clearly identified with codes or names in order to avoid misinterpretation of results. When the kit is used for the screening of blood units, bar code labeling and electronic reading is strongly recommended. 4-Hemolysed and visibly hyperlipemic (milky) samples have to be discarded as they could generate false results. Samples containing residues of fibrin or heavy particles or microbial filaments and bodies should be discarded as they could give rise to false results.

5-Sera and plasma can be stored at $+2-8^{\circ} \mathrm{C}$ for up to seven days after collection. For longer storage periods, samples can be stored frozen at $-20^{\circ} \mathrm{C}$ for several months. Any frozen samples should not be frozen/thawed more than once as this may generate particles that could affect the test result.

6-If particles are present, centrifuge at $2.000 \mathrm{rpm}$ for 20 min or filter using $0.2-0.8 \mathrm{u}$ filters to claen up the sample for testing. Protocol was approved from local ethical committee in Beni-Suef University. 


\section{RESULTS}

This study is a retrospective observational study and it was carried out on 30055 blood donors in the blood bank of Beni-Suef University Hospital in the period between November 2007 and April 2015.

In the present study, the age of the studied group ranged from 18 to 65 years with a mean and SD (29.92 \pm 7.65 years). About (28197) of the donors (93.3\%) were males and (1858) of the donors $(6.7 \%)$ were females. We found that donors with blood group (A ) positive were representing the most common donors with percentage of $(32.9 \%)$ followed by donors with group ( O ) positive with percentage of $(26 \%)$.

\section{A-Serology of donors:}

The percentage of donors with HCV Ab were $9.4 \%$ (2835), the percentage of donors with HBsAg were $1.6 \%$ (491), the percentage of donors with positive HIV Ab were $0.1 \%$ ( 18 ) and the percentage of donors with positive VDRL of syphilis were nearly $0.0 \%(7)$ (Table 1$)$.

\section{A) Descriptive data of $\mathrm{HCV}$ positive donor:}

The mean age of positive $\mathrm{HCV}$ Ab group was older than negative group $(32 \pm 8.5$ Vs $29.69 \pm 7.5)$ with $P$ value (0.043) (Table 2). Most of positive HCV group were males (2646 of 2835) representing (93.3\%) of positive HCV
Ab with $P$ value of $(0.001)$ (Table 1$)$.

\section{B) Descriptive data of positive $\mathrm{HBV}$ donor:}

The mean age of positive HBV blood donors was significantly older than negative HBV group $(30.09 \pm 7.7 \mathrm{Vs} 29.9 \pm 7.6)$ with $P$ value (0.046) (Table 3). There was no significant difference regarding $\mathrm{CBC}$ in both groups of donors as shown in the following table (Table 4).

\section{C) Descriptive data of positive HIV donor:}

The present study found that the mean age of positive HIV blood donors was nearly the same of negative HIV group (29.22 \pm 6.8 Vs $29.92 \pm 7.6)$ with $P$ value $(0.071)$ (Table 4) Regarding blood pressure of donors there was no significant difference between mean of Systolic blood pressure in both group (118.89 \pm 3.2 Vs $119.97 \pm 2.9$, respectively) with $P$ value of (0.080). Also, there was no significant difference between both group regarding diastolic blood pressure $(81.67 \pm 3.8$ Vs $79.81 \pm 2.3$, respectively) with $P$ value of $(0.056)$ (Table 5) There was no significant difference regarding $\mathrm{CBC}$ in both groups of donors as shown in the following table (Table 7).

\section{D) Descriptive data of Positive Syphilis donors:}

The mean age of positive Syphilis blood donors was significantly older than negative Syphilis group $(31.71 \pm 7.8 \mathrm{Vs}$ $29.92 \pm 7.6$, respectively) with $P$ value $(0.044)$

Table 1: Serology and blood group of blood bank donors.

\begin{tabular}{lcc}
\hline Serology & Number & Percentage \\
\hline HCV Ab & 2835 & 9.4 \\
HBsAg & 491 & 0.1 \\
HIV Ab & 18 & 0.0002 \\
Syphilis VDRL & 7 & Percentage \\
Blood group & Number & 32.9 \\
A positive & 9900 & 26 \\
O positive & 7813 & 23.9 \\
B positive & 7188 & 10.1 \\
AB positive & 3022 & 2.6 \\
A negative & 778 & 2.1 \\
O negative & 629 & 1.7 \\
B negative & 515 & 0.7 \\
AB negative & 2100 & 2 \\
\hline
\end{tabular}


Table 2: Descriptive data of HCV positive donor.

\begin{tabular}{lc}
\hline Age & Mean \pm SD \\
\hline Positive HCV Ab & $32 \pm 8.5$ \\
Negative HCV Ab & $29.69 \pm 7.5$ \\
\hline
\end{tabular}

Table 3: Descriptive data of positive HBV donor

\begin{tabular}{lccc}
\hline & Positive HBV (491) & Negative HBV (29564) & \\
\hline Male & 459 & 27738 & \\
Female & 32 & 1826 & 0.001 \\
\hline
\end{tabular}

Table 4: Comparison regarding gender in both Hepatitis donor group and Comparison Regarding residence in both groups (Positive HIV donors)

\begin{tabular}{lcc}
\hline Gender & Positive HIV (18) & Negative HIV (29564) \\
\hline Male & 17 & 28180 \\
Female & 1 & 1857 \\
Residence & Negative HIV value & Positive HIV \\
Urban Area & $12774(42.5 \%)$ & $8(44.5 \%)$ \\
Rural Area & $17263(57.5 \%)$ & $10(55.5 \%)$ \\
\hline
\end{tabular}

Table 5: Comparison according to BP of both group (Positive HIV donors)

\begin{tabular}{lccc}
\hline & Positive HIV & Negative HIV & P value \\
\hline SBP & $118.8 \pm 3.2$ & $119.9 \pm 2.9$ & 0.080 \\
DBP & $81.6 \pm 3.8$ & $79.8 \pm 2.3$ & 0.056 \\
\hline
\end{tabular}

Table 6: Comparison according to age of both group (Positive Syphilis donors)

\begin{tabular}{lcc}
\hline Age & Mean \pm SD & P value \\
\hline Positive VDRL & $31.71 \pm 7.8$ & 0.044 \\
Negative VDRL & $29.92 \pm 7.6$ \\
\hline
\end{tabular}


Table 7: Comparison according to CBC of both group (Positive HIV donors)

\begin{tabular}{lccc}
\hline & Positive HIV & HIV Negative & P value \\
\hline HB & $14.46 \pm 1.2$ & $14.38 \pm 1.3$ & 0.081 \\
TLC & $6.25 \pm 1.8$ & $7.3 \pm 4.5$ & 0.076 \\
PLT & & & \\
\hline
\end{tabular}

\section{DISCUSSION}

Infection with HBV , HCV , HIV and syphilis is a worldwide significant problem in public health. In general, the diagnosis of HBV, HCV, HIV and syphilis is based on the presence of the corresponding antigens or antibodies in blood serum. Prevalence of HBV, HCV, HIV and syphilis was dependent on many factors, such as number of infected persons in the family, prevalence of infection in the area the subject lives, frequency of the disease in the neighboring countries, rate of immigration, variations in geographical distribution as well as population differences in terms of lifestyle, awareness, sensitivity and specificity of tests used, donor selection criteria ${ }^{[7]}$.

In this study, the prevalence of Hepatitis B infection obtained was $(1.6 \%)$. In concordance to our study, nearly similar frequency rates of HBV have been reported in Suez Canal University Blood bank study $(2.3 \%)^{[8]}$. In same context to our study, other studies that were conducted in different countries showed nearly similar prevelance as those in Iran $(2.3 \%)^{[9]}$. On the other hand, the seroprevalence of HBV was higher in Ghana $(15 \%)^{[10]}$.

According to the $\mathrm{WHO}$, the world wide prevalence with $\mathrm{HCV}$ is $3 \%$, representing 170 million chronic carriers. The highest prevalence was in Africa, (5.3\%), whereas the lowest prevalence was in Europe , $(1.03 \%)$. Egypt has a very high prevalence of antibody against hepatitis $\mathrm{C}$ virus (HCV) resulting in a high morbidity and mortality from chronic liver disease, cirrhosis, and hepatocellular carcinoma. Around $20 \%$ of blood donors are seropositive by ELISA for antibodies to HCV. Children have lower rates of disease, but prevalence rises steeply with age $\mathrm{e}^{[11]}$.

This study showed that prevalence of hepatitis C infection was $(9.4 \%)$. In concordance to our study nearly similar rates were reported in other studies that conducted in different countries as in Pakistan $(8.1 \%)^{[12]}$, in Turkey $(5.2 \%)^{[13]}$. On other hand, lower rates for HCV prevalence has been reported in in French $(0.68 \%)^{[14]}$. The high prevalence of hepatitis $\mathrm{C}$ infections in Egypt serves as a sentinel warning for public health professionals.
This study showed that prevelance of HIV was $(0.1 \%)$, in concordanceto our study nearly similar rates were reported in other in Suez Canal blood bank study that showed a prevelance of HIV was $(0 \%)^{(8)}$. On other hand seroprevalence of HIV in blood donors was higher in Ghana $(3.8 \%)^{[10]}$.

In this study, the prevalence of syphilis was found to be $(0.0002 \%)$. In the same context to our study nearly similar rates were reported in Egyptian studies as those conducted in Suez Canal blood bank study that showed a low prevelance of Syphilis $(0 \%)^{[8]}$, and Cairo university blood bank study $(0.13 \%)^{[6]}$, this was consistent with the observed low prevalence of syphilis seroprevalence in the general population in Egypt because most of the modes of transmission are not found in this Islamic oriental country. On other hand higher seroprevalence rates were found in Ghana $(7.5 \%)^{[15]}$, and in Ethiopia $(12.8 \%)^{[16]}$.

In this study, the majority of donors 28197 (93.3\%) were males, while females were 1858 (6.7\%). Prevalence of HBsAg and anti-HCV were significantly higher among males $(1.5 \%, 8.8 \%$, respectively) than females $(0.1 \%$ and $0.6 \%$, respectively) with $(P<0.001)$. In Egypt women are usually housewives and this may lead them to avoid outdoor activities so fewer females donation made them fewer screened than males. This also ,agree with Suez Canal blood bank study which was conducted in a period between 1996 to 2011 and showed that majority of donors, 125,562 (84\%), were males, compared to $23,819(16 \%)$ females. Prevalence of HBsAg and anti-HCV were significantly higher among males $(2.3 \%$ and $7.3 \%$, respectively) than females $(2.1 \%, 6.6 \%$, respectively) $(P<0.0001)^{[8]}$.

This study showed that blood donors from the urban area represent about $(42.5 \%)$ of all donors and these percentage was little than those from the rural area, and also , there was a significantly higher prevalence of both HBsAg and anti-HCV prevalence among rural populations (1.9\% and $7.3 \%$, respectively) compared with urban populations $(1.4 \%$ and $6.1 \%$, respectively) $(P<0.001)$. This also agreed with Suez Canal blood bank study that showed there was a significantly higher 
prevalence of both $\mathrm{HBsAg}$ and anti-HCV prevalence among rural populations $(2.6 \%$ and $7.9 \%$, respectively) compared with urban populations ( $2 \%$ and $6.6 \%$, respectively) $(P<.0001)^{[8]}$.

According to the findings of this study, there was a decreasing prevalence of $\mathrm{HCV}$ among blood donors as the annual anti-HCV prevalence dropped significantly from 9.4\% (2007- 2008) to $3.2 \%$ in (2014- 2015) $(P<0.01)$, whereas there was a mild variation in the prevalence of HBsAg, throughout the study period, that was not statistically significant $(\mathrm{P}=0.6)$. This also, agreed with Suez Canal blood bank study that showed that there was a decreasing prevalence of $\mathrm{HCV}$ as the annual Anti-HCV prevalence estimates dropped significantly $(P<.0001)$ from $14.9 \%$ (1996) to $3.5 \%$ (2011) whereas there was a mild variation in the prevalence of HBsAg, throughout the study period, that was not statistically significant $(P>$.05).( Nada et al .2013), study that showed a consistent steady decline in the seropositivity for HCV antibody was observed ${ }^{[8]}$

The declining trends in seroprevalence of $\mathrm{HCV}$ in the blood donors is a good signal as the risk of acquiring infections due to transfusion is decreased. This declining trend may be due to more public awareness about the disease, the use of newer generation kits with improvements in specificity and sensitivity of blood-borne pathogen detection and larger sample size due to increased donors' recruitment efforts leading to more accurate results.

\section{CONFLICT OF INTEREST}

There are no conflicts of interest.

\section{REFERENCES}

1. Hatzimouratidis K, Eardley I, Giuliano F, Moncada I and Salonia A (2014): Guidelines on Male Sexual Dysfunction: Erectile dysfunction and premature ejaculation, European Association of Urology; 6-35.

2. Seyam R, Albakry A, Ghobish A, Arif H, Dandash K and Rashwan H (2003): Prevalence of erectile dysfunction and its correlates in Egypt: a community based study. Int J Impot Res; 15 (4):237-245.

3. Björndahl L, Aleksander G, Herman $\mathrm{T}$ and Weidner W, (2010): Clinical Andrology (EAU/ESAU Course Guidelines, page 380).

4. Goldstein I, Newman L and Baum N, (1998): Safety and efficacy outcome of Mentor alpha-1 inflatable penile prosthesis implantation for impotence treatment. J Urol; 157: 833-9.

5. Hellstrom W, (2007): Current safety and tolerability issues in men with erectile dysfunction receiving PDE5 inhibitors. Int J Clin Pract.; 61: 1547-1554.

6. Ronald L, Carol J, William D, William H, Stanley E, Vera J and Richard L, (2001): patient and partner satisfaction with Viagra (sildenafil citrate) treatment as determined by the erectile dysfunction inventory of treatment satisfaction questionnaire. Urology, 57(5), 960-965.

7. Bettocchi C, Palumbo F, Spilotros M, Lucarelli G, Ricapito V, Palazzo S, Battaglia M, Selvaggi F and Ditonno P, (2009): Penile prosthesis implant : when, what and how.JMH;6:299-306.

8. Minervini A, Ralph D and Pryor J, (2005): Outcome of penile prosthesis implantation for treating erectile dysfunction: experience with 504 procedures. BJU Int; 97: 129-33.

9. Natali A, Olianas R and Fisch M, (2008): Penile implantation in Europe: successes and complications with 253 implants in Italy and Germany. J Sex Med; 5(6):1503-12.

10. Bhojwani A, Jain R, Kockelbergh R and Terry $\mathrm{T}$, (1998): Sexual satisfaction after penile prosthesis insertion for the treatment of erectile dysfunction. Sex Dysfunct; 1:133-6.

11. Halloway F and Farah R, (1997): Intermediate term assessment of the reliability, function and patient satisfaction with the AMS700 Ultrex penile prostheses. J Urol; 157: 1687-91.

12. Stanley E, Eric W, Stephen B, Frederic L, Arthur L, Kevin M, Vera S and Andallen D, (1999): EDITS: development of questionnaires for evaluating satisfaction with treatments for erectile dysfunction. Urology 53:793-799.

13. Humberto G and LeRoy J, (2012): Outcomes of and Satisfaction with the Inflatable Penile Prosthesis in the Elderly Male. Advances in Urology, Article ID 240963, 4pages.

14. Vakalopoulos I, Kampantais S, Ioannidis S, Laskaridis L, Dimopoulos P, Toutziaris C, Koptsis M, Henry G and Katsikas V, (2013): High Patient Satisfaction after Inflatable Penile Prostheses Implantation Correlates with Female Partner Satisfaction J Sex Med 2013; Nov; 10 (11):2774-81. 